RESEARCH ARTICLE

\title{
Risk Factors Associated with Colonization of Intestine by Carbapenem Resistant Enterobacteriaceae in a Pediatric ICU
}

\author{
Sonal Saxena ${ }^{1}$, Suresh Kumar ${ }^{2}$, Virender Kumar ${ }^{3}$, Ravinder Kaur ${ }^{1}$ \\ ${ }^{1}$ Department of Microbiology, Lady Hardinge Medical College, New Delhi, India \\ ${ }^{2}$ Department of Microbiology, Moolchand Medcity Hospital, New Delhi, India \\ ${ }^{3}$ Department of Pediatrics, Kalawati Saran Children Hospital, Lady Hardinge Medical College, New Delhi, India
}

\begin{abstract}
Objectives: Asymptomatic colonization of the gastrointestinal tract by Carbapenem Resistant Enterobacteriaceae (CRE) constitutes a reservoir for transmission that may remain unidentified in hospitals that do not implement active surveillance testing. Aim: To assess the magnitude of colonization of carbapenem resistant Enterobacteriaceae and to analyze various risk factors associated with this colonization in children admitted in PICU.
\end{abstract}

Methods: Three-hundred patients admitted to Pediatric Intensive Care Unit (ICU) which tested negative for CRE colonization upon admission were studied for colonization of intestine by CRE after two days of stay in ICU. Risk factors were analyzed with respect to CRE colonized and non CRE colonized groups.

Results: Thirty-two (10.67\%) patients were found to be colonized with CRE; Klebsiella pneumoniae being the predominant species followed by Escherichia coli using CDC screening method. Univariate analysis of risk factors revealed acute bronchopneumonia, acute febrile illness, Gullian-Barre syndrome to be significantly associated with colonization with CRE. Also, sepsis, presence of central line, mechanical ventilation, protein energy malnutrition and intake of steroids, Piperacillin-Tazobactam was found to be highly significant $(p<0.001)$. The odds of developing colonization were significantly higher in children with renal and respiratory disease, sepsis and with intake of broadspectrum antibiotics.

Conclusions: Antimicrobial resistance is a huge public health problem in developing countries and these colonized patients can act as reservoirs of multi drug resistant bacteria resulting in untreatable healthcare associated infections. Large scale epidemiological surveillance studies of CRE using affordable phenotypic methods along with stringent infection prevention control strategies are required. J Microbiol Infect Dis 2020; 10(2):75-81.

Keywords: Carbapenem-Resistant Enterobacteriaceae, CRE, Colonization, Risk factors

\section{INTRODUCTION}

In the era of antimicrobial resistance drugs belonging to carbapenem category are considered as drug of choice against multi drug resistant organisms especially in the hospital settings. However, the past few years have seen resistance developing to this class of drugs as well. Carbapenem-resistant Enterobacteriaceae (CRE) has emerged as a cause of healthcare infections in several regions around the world. They are now considered an important public health threat classified as urgent by the Centre for Disease Control and Prevention [1,2]. These isolates have a wide, geographical dissemination and limited therapeutic alternatives and are associated with high morbidity and mortality [3,4].

Centers for Disease Control and Prevention revised the definition of CRE in 2015 as the organisms that are nonsusceptible to imipenem, meropenem, doripenem, or ertapenem or a documented isolate that possesses a carbapenamase (KPC) [5]. Asymptomatic colonization of the gastrointestinal tract by CRE constitutes a reservoir for transmission that may remain unidentified in hospitals that do not 
implement active surveillance testing [2,6]. Factors associated with CRE fecal carriage may include antibiotic exposure, malignancy, nonsurgical invasive procedures, prolonged hospital stay, admission to intensive care units (ICU) and sharing a room with high known carriers [7]. Colonization is considered to be a prerequisite for developing infections [8]. A systematic review analysed, 1806 initially CRE colonized patients. Of these, 299 clinical infections were observed for a cumulative rate of $16.5 \%$ [9].

Most colonization studies are retrospective and were conducted in areas with high prevalence of KPC or New Delhi Metallo- $\beta$-lactamase (NDM) carrying strains of Klebsiella pneumoniae or in outbreak situations; therefore, these findings may not apply to other settings [7]. The objective of this study was to assess the magnitude of colonization of carbapenem resistant Enterobacteriaceae and to assess the various risk factors associated with this colonization in children admitted in PICU.

\section{METHODS}

The study was a prospective observational cross section study conducted at Pediatric ICU (PICU) of Kalawati Saran Children Hospital and Department of Microbiology, Lady Hardinge Medical College and, New Delhi. The study was conducted over a period of 18 months. The sample size was calculated using data obtained from pilot study done earlier at our center. Keeping time constraints and limited resources in mind the sample size was calculated at 300 . The study protocol was approved by the institutional ethics committee.

All patients admitted in Pediatric ICU assenting/consenting to be part of study were included with the following Exclusion criteria:

1) Patients transferred from other hospitals to PICU and those whose history of hospitalization/antibiotic intake could not be elicited.

2) Patients whose rectal swab taken at time of admission yielded CRE.

300 patients fulfilling the inclusion and exclusion criteria were included in the study. Patient's details were noted in a structured pretested performa after written informed consent and assent where ever applicable (as per ethical guidelines) was taken from the parents/child. The detailed history included demographic details, presenting signs and symptoms, antibiotic therapy etc. Risk factor details such as underlying medical condition, history of any surgery done, immune status, history of steroid therapy, nutrition status, and presence of any devices like intravenous or CVP catheter, urinary catheter, history of antibiotic intake with duration was also noted. Risk factors were analyzed with respect to CRE colonized and non CRE colonized groups.

Microbiology: Two rectal swabs were collected from each patient, one at the time of admission and another after two days of admission. Two swabs were collected from each patient on each occasion. One swab was kept in a tube containing Trypticase Soya broth containing 10 $\mu \mathrm{g}$ of Meropenem disc as per CDC method [10] to detect CRE. The second swab was streaked on MacConkey \& XLD agar as per routine processing protocol. Next day the tube was macroscopically observed for turbidity. A loopful of growth was inoculated on MacConkey agar and was incubated overnight aerobically at 37 ${ }^{0} \mathrm{C}$. Next day this plate was observed for any visible growth. For no growth and non-lactose fermenting colonies, no further processing was done. Identification of all bacterial isolates was done by vitek $2^{\mathrm{TM}}$. Lactose fermenting colonies identified as $E$. coli and $K$. pneumoniae were subjected to antibiotic susceptibility testing as per CLSI guidelines using Kirby Bauer disc diffusion method [11] to the following antibiotics amikacin $30 \mu \mathrm{g}$, ceftazidime $30 \mu \mathrm{g}$, piperacillin tazobactam 100/10 $\mu \mathrm{g}$, amoxycillin clavulanate $20 / 10 \mu \mathrm{g}$, ciprofloxacin $5 \mu \mathrm{g}$, gentamicin $10 \mu \mathrm{g}$, imipenem $10 \mu \mathrm{g}$, meropenem $10 \mu \mathrm{g}$, ceftazidime clavulanic acid 30/10 $\mu \mathrm{g}$. All strains were tested for the production of KPCs by Modified Hodge Test [11]. MIC was done using E-Test (Bio Merieux, France) as per standard procedure [11].

\section{Statistical analysis}

The antimicrobial susceptibility data was analyzed using WHONET@ software. The data was statistically analyzed by using SPSS version 12

\section{RESULTS}

A total of 300 patients were included in the study. Among these, there was slight preponderance of 157 (52.33\%) male patients 
over 143 (47.67\%) female patients. Mean age of patients admitted in PICU included in the study was 3.2 with +2.9 years. Upon culture 152 organisms were isolated from 300 rectal swabs. Table 1 gives distribution of various organisms isolated from those swabs. Commonest organism isolated was Klebsiella species, which was isolated from 51 swabs followed by Escherichia coli (32 swabs). The patients from whom CRE was isolated were included in CRE group while the rest formed the non CRE (NCRE) group.

\section{CRE group}

A total of 32 (10.67\%) isolates of carbapenem resistant Enterobacteriaceae were isolated using CDC screening method. Of these 32 isolates 20 $(62.5 \%)$ were identified as $K$. pneumoniae while $12(37.5 \%)$ were identified as Escherichia coli. All isolates of CRE were further subjected to MIC determination by $E$ test. All isolates were found resistant with Meropenem with mean MIC of $5.8+8.2$, MIC50 of 4 and MIC90 of 8 . These 32 isolates were also subjected to Modified Hodge Test. MHT was positive only in 19 cases $(59.38 \%)$ suggesting mechanisms other than KPC production.

The antibiotic resistance profile of these 32 isolates is shown in Table 2. Seven Escherichia coli and sixteen isolates of $K$. pneumoniae were resistant to imipenem by disc diffusion. Also $8 E$. coli $(66.67 \%)$ and 16 K. pneumoniae (80\%) among 32 isolates were resistant to more than 5 antibiotics leaving very few therapeutic options.

\section{Non- CRE (NCRE) Group}

One-hundred-twenty isolates were obtained from 268 patients included in this group.

Univariate analysis of risk factors revealed acute bronchopneumonia, acute febrile illness, Guillain Barre Syndrome to be significantly associated with colonization with CRE. Also, sepsis, presence of central line, mechanical ventilation, protein energy malnutrition and intake of steroids, piperacillin tazobactam was found to be highly significant $(p<0.001)$ as shown in Table 3.

Multivariate logistic regression was done to analyze the risk factors in terms of odds ratio to the development of colonization with CRE. The odds of developing colonization were significantly higher in children with respiratory disease with $p$ value of 0.005 (1.4-14.8), sepsis with $p$ value of $<0.001$ (1.7-7.1) and with intake of broad-spectrum antibiotics such as ceftriaxone ( $\mathrm{p}$ value of 0.076 ; Cl: $0.8-3.5$ ).

Table 1. Distribution of various organisms from all patients.

\begin{tabular}{lccc}
\hline Variables & NCRE & CRE & Total \\
\hline E. coli & 20 & 12 & 32 \\
Klebsiella & 31 & 20 & 51 \\
Pseudomonas & 26 & 0 & 26 \\
Acinetobacter & 32 & 0 & 32 \\
Proteus & 11 & 0 & 11 \\
Total & 120 & 32 & 152 \\
\hline
\end{tabular}

CRE $=$ Carbapenem Resistant Enterobacteriaceae,

$\mathrm{NCRE}=$ Non Carbapenem Resistant Enterobacteriaceae

Table 2. Antibiotic Resistance profile of Carbapenem Resistant Enterobacteriaceae Isolates.

\begin{tabular}{ccc}
\hline Variables & E. coli 12(\%) & $\begin{array}{c}\text { K. pneumoniae 20 } \\
(\%)\end{array}$ \\
\hline Amikacin & $2(16.67)$ & $12(60)$ \\
Ciprofloxacin & $7(58.34)$ & $16(80)$ \\
Imipenem & $7(58.34)$ & $16(80)$ \\
Meropenem & $12(100)$ & $20(100)$ \\
Ceftazidime & $2(16.67)$ & $14(70)$ \\
Piperacillin/ & $8(66.67)$ & $11(55)$ \\
Tazobactam & & \\
Amoxycillin/ & $2(16.67)$ & $14(70)$ \\
Clavulanic acid & & $16(80)$ \\
Gentamicin & $5(41.67)$ & \\
\hline
\end{tabular}

\section{DISCUSSION}

A total of 300 patients were screened for gut colonization using rectal swabs Rectal swabs have been reported to be more sensitive than perianal swabs by Wiener-Well et al [12]. Many authors have used chrome agar or selective agar with antibiotics for detection of CRE [9,1315]. Various methods have been used by authors from India [16-18]. We found CDC method to be easy, practical and cost effective.

Few studies have reported gut colonization by CRE in non-outbreak conditions. A study from Pakistan has reported fecal carriage of $18.5 \%$ while Turner et al from Cambodia reported CRE colonization rate at $7.5 \%$ in infants $[13,19]$. Tran et al from Vietnam have reported a high colonization rate of $32 \%$ on day 2 of hospitalization [14]. 
Table 3. Risk factor analysis of Colonized Vs Non Colonized patients.

\begin{tabular}{|c|c|c|c|}
\hline Diagnosis & $\begin{array}{c}\text { CRE (n=32) } \\
N(\%)\end{array}$ & $\begin{array}{c}\text { NCRE (268) } \\
\text { N (\%) }\end{array}$ & $P$ value \\
\hline Acute bronchopneumonia & $18(56.25)$ & $91(33.96)$ & 0.007 \\
\hline AGE & $6(18.75)$ & $36(13.43)$ & 0.206 \\
\hline Sepsis & $18(56.25)$ & $72(26.87)$ & $<0.001$ \\
\hline Acute febrile illness & $8(25.00)$ & $36(13.43)$ & 0.040 \\
\hline Ascites & $3(9.38)$ & $16(5.97)$ & 0.227 \\
\hline Dengue/Chikungunya & $1(3.13)$ & $16(5.97)$ & 0.255 \\
\hline Gullian Barre Syndrome & $2(6.25)$ & $4(1.49)$ & 0.035 \\
\hline Malaria & $1(3.13)$ & $13(4.85)$ & 0.331 \\
\hline Meningitis & $2(6.25)$ & $24(8.96)$ & 0.304 \\
\hline Seizure disorder & $0(0.00)$ & $13(4.85)$ & 0.101 \\
\hline Hepatitis & $4(12.5)$ & $19(7.09)$ & 0.138 \\
\hline Grade 1 & $22(68.75)$ & $44(16.42)$ & $<0.001$ \\
\hline Grade 2 & $5(15.63)$ & $8(2.99)$ & $<0.001$ \\
\hline Steroid Therapy & $17(53.13)$ & $49(18.28)$ & $<0.001$ \\
\hline Other underlying conditions & $6(18.75)$ & $27(10.07)$ & 0.069 \\
\hline Renal & $2(6.25)$ & $3(1.12)$ & 0.016 \\
\hline Respiratory & $4(12.5)$ & $8(2.99)$ & 0.005 \\
\hline Endocrinal & $1(3.13)$ & $5(1.87)$ & 0.315 \\
\hline GIT & $0(0.00)$ & $1(0.37)$ & 0.365 \\
\hline Musculoskeletal & $0(0.00)$ & $3(1.12)$ & 0.274 \\
\hline Urinary catheter & $31(96.88)$ & $252(94.03)$ & 0.255 \\
\hline Intravenous Cannula & $32(100.00)$ & $266(99.25)$ & 0.312 \\
\hline CVP line & $29(90.63)$ & $103(38.43)$ & $<0.001$ \\
\hline Mechanical Ventilation & $20(62.5)$ & $29(10.82)$ & $<0.001$ \\
\hline \multicolumn{4}{|l|}{ H/O Antibiotic Intake } \\
\hline Amoxycillin-Clavulanic acid & $9(28.13)$ & $36(13.43)$ & 0.014 \\
\hline Amikacin & $15(46.88)$ & $76(28.36)$ & 0.016 \\
\hline Piperacillin Tazobactam & $4(12.50)$ & $0(0.00)$ & $<0.001$ \\
\hline Vancomycin & $12(37.5)$ & $98(36.57)$ & 0.459 \\
\hline Meropenem & $10(31.25)$ & $90(33.58)$ & 0.396 \\
\hline Colistin & $5(15.63)$ & $37(13.81)$ & 0.390 \\
\hline Metronidazole & $6(18.75)$ & $30(11.19)$ & 0.107 \\
\hline Ceftriaxone & $17(53.13)$ & 107 (39.93) & 0.076 \\
\hline
\end{tabular}

$\mathrm{CR} \overline{\mathrm{E}}=$ Carbapenem Resistant Enterobacteriaceae, $\mathrm{NCRE}=$ Non Carbapenem Resistant Enterobacteriaceae

From India two studies have reported similar rates. Mittal et al from Delhi have reported colonization rates of $11 \%$ (on day 1 ) and $22 \%$ (on day 4). They also reported $6.6 \%$ colonization rate amongst non-hospitalized patients [7]. Since our study included only those patients whose initial culture on admission was negative 
for CRE, we cannot comment on initial colonization. A study from Delhi in 2014 has reported colonization rate of $9.9 \%$ among outpatients in an urban area [20]. As expected higher colonization rates have been reported in outbreak situations indicating the epidemic potential of these organisms [14,21]. A point prevalence survey for screening for colonization at 12 Vietnamese hospitals found $52 \%$ patients colonized with CRE [14]. Gonzales et al from Mexico found the prevalence of CRE at $10.9 \%$ during a CRE outbreak (21]. The children in our study were swabbed only once at 48 hours, so it is possible that a greater number of children could have become colonized with passage of time but were missed. This could explain relatively lower rate of isolation but the purpose in our study was to find out how many children develop colonization after 48 hours which is recommended for definition of healthcare associated infections to develop.

K. pneumoniae was found to be the predominant colonizer in our patients followed by Escherichia coli. Tran et al from Vietnam also reported Klebsiella as predominant species (69\%) followed by Escherichia coli (58\%) [14). A study from India also found $58 \%$ Klebsiella amongst CRE colonizers [16,18]. A meta-analysis of 61 studies reported $E$. coli and Klebsiella as predominant Enterobactericae members with carbapenem resistance in the Asian region [1]. Other organisms such as Enterobacter species, Proteus species and Citrobacter have also been reported [22-24]. But those studies mostly deal with clinical isolates and not with colonization.

K. pneumoniae showed high level of resistance as compared to E. coli. This outcome is not surprising as Klebsiella species is not only leading cause of nosocomial infection in PICU, but also a notorious collector of multi drug resistance plasmidsCRE are known to carry multiple resistance determinants \& poly antimicrobial resistance is common in these isolates. Multi drug resistance has been reported in other studies as well as from India leaving few therapeutic options for treatment [7].

Higher resistance was found for meropenem as compared to imipenem. This discrepant pattern is a common finding in many studies $[25,26]$. Differences in mechanism of resistance such as expression of beta lactamases, porin loss, efflux pumps or loss/alteration in penicillin binding protein may contribute to this variability [26]. In our settings MHT detected $59.3 \%$ strains to possess KPC production activity. A study from USA found only $50 \%$ isolates to demonstrate KPC [24]. Another study from India reported only $18 \%$ isolates positive by MHT clearly demonstrating multiple mechanisms of action against carbapenems [18]. This highlights the importance of performing additional screening tests such as Carba NP, metallo-beta-lactamase detection or molecular analysis to accurately detect the susceptibility to carbapenems. However, presence of MBL is known to demonstrate negative or weakly positive MHT results. Since we could not analyze these isolates for MBL or perform PCR for specific genes, this could be a limiting factor in our study.

In our study all colonized patients were prescribed one or more antibiotics. This is expected knowing the life-threatening nature of illnesses in ICUs. Logan in meta-analysis has reported $97 \%$ of children had received antibiotics before CRE infection [25]. Prior use of antibiotics tends to inhibit susceptible normal flora in the gut leading to colonization with multi drug resistant organisms such as CRE. In our study exposure to beta lactams and beta lactam inhibitors (amoxyclavulanate and piperacillintazobacatam) were found to be significantly associated with colonization. Similar to our findings a study from Pakistan reported amoxycillin clavulanate as a significant risk factor for CRE colonization with NDM [19]. A Mexican study in 2015 found significant association of CRE colonization with intake of piperacillin tazobactam and ciprofloxacin [21).

Logan et al in 2012 noted pulmonary disease followed by pre-maturity and small bowel disease to be associated with CRE in $92 \%$ of cases [25]. Univariate analysis in a multi- center study from Vietnam in 2019 found stay in hospital, pulmonary disease, healthcare associated sepsis, and intubation, treatment with carbapenem and colistin and fluoroquinolones to be significant risk factors for colonization [14]. Mittal et al from Delhi found ICU stay, ventilator, therapy with aminoglycosides and carbapenem to be significant risk factors in univariant analysis [7]. Borer et al from Israel identified the antibiotic therapy such as aminopenicillins, 
being bedridden, and nursing home residency as predictors for CRE colonization [27]. A study from Greece identified male gender, prior bed occupants, and patients in nearby beds colonized with CRE, tracheotomy, number of invasive catheters inserted, and number of antibiotics administered as the major risk factors for KPC producing $K$. pneumoniae colonization [28]. Multiple researchers have reported immunosuppression, increased length of stay, antibiotic intake and ventilation as risk factors ffor colnization [7,14,16,21,24,25].

Colonization is considered to be a prerequisite for infection, which suggest that prevention of CRE colonization is important in preventing the morbidity and mortality associated with these infections. Limitations of study: Molecular analysis of the isolates could not be carried out due to resource constraints. This could be a limiting factor as the molecular epidemiology of these strains could not be determined. Secondly, the patients were swabbed only once after admission for colonization so it is possible that a greater number of children could have become colonized with passage of time which have been missed in our study.

\section{Conclusion}

Our point prevalence study demonstrates the colonization and risk factors attributing to the colonization with CRE in an ICU setting. It is important that in an era when antimicrobial resistance is a huge public health problem these colonized patients can act as reservoirs of these multi drug resistant bacteria. In order to understand the magnitude of this problem, large scale epidemiological surveillance studies of CRE using affordable phenotypic methods are required. This problem assessment can prompt antimicrobial stewardship along with stringent infection prevention control strategies to control this problem.

\section{REFERENCES}

1. $X u Y, G u B$, Huang $M$, et al. Epidemiology of carbapenem resistant Enterobacteriaceae (CRE) during 2000-2012 in Asia. J Thorac Dis 2015; 7(3):376-385.

2. Litzow JM, Gill CJ, Mantaring JB, et al. High frequency of multidrug-resistant gram-negative rods in 2 neonatal intensive care units in the Philippines. Infect Control Hosp Epidemiol 2009; 30:543-549.
3. Schwaber MJ, Klarfeld-Lidji S, Navon-Venezia S, Schwartz D, Leavitt A, Carmeli Y. Predictors of carbapenem-resistant Klebsiella pneumoniae acquisition among hospitalized adults and effect of acquisition on mortality Antimicrob Agents Chemother 2008; 52:1028-1033.

4. Borer A, Saidel-Odes L, Riesenberg K, et al. Attributable mortality rate for carbapenemresistant Klebsiella pneumoniae bacteremia. Infect Control Hosp Epidemiol 2009; 30:972-6.

5. Carbapenem resistant Enterobacteriaceae: CRE definition. Available from: http://www.cdc.gov/hai/organisms/cre/definition.ht $\mathrm{ml}$ [Accessed June 15, 2019]

6. Maamar E, Ferjani S, Jendoubi A, et al. High Prevalence of Gut Microbiota Colonization with Broad-Spectrum Cephalosporin Resistant Enterobacteriaceae in a Tunisian Intensive Care Unit. Front Microbiol 2016; 7:1859.

7. Mittal G, Gaind R, Kumar D, et al. Risk factors for fecal carriage of carbapenemase producing Enterobacteriaceae among intensive care unit patients from a tertiary care center in India BMC Microbiol 2016;16:138.

8. Bonten MJ, Weinstein RA. The role of colonization in the pathogenesis of nosocomial infections. Infect Control Hosp Epidemiol 1996; 17:193-200.

9. Tischendorf J, Almeida de Avila R, Nasia Safdar N. Risk of infection following colonization with carbapenem- resistant Enterobactericeae: A systematic review. Am J Infect Control 2016; 44(5): 539-543.

10. Center for Disease Control and Prevention. Laboratory Protocol for Detection of CarbapenemResistant or Carbapenemase-Producing, Klebsiella spp. and E. coli from Rectal Swabs. Available https://www.cdc.gov/HAl/pdfs/labSettings/Klebsiell a_or_Ecoli.pdf.

11. CLSI. Performance Standards for Antimicrobial Susceptibility Testing. 27th ed. CLSI supplement M100. Wayne, PA: Clinical and Laboratory Standards Institute; 2017.

12. Wiener-Well Y, Rudensky B, Yinnon AM, et al. Carriage rate of carbapenem-resistant Klebsiella pneumoniae in hospitalized patients during a national outbreak. J Hosp Infect 2010; 74:344349.

13. Turner P, Pol S, Soeng S, et al. Turner C High Prevalence of Antimicrobial-resistant Gramnegative Colonization in Hospitalized Cambodian Infants. Pediatric Infect Dis J 2016; 35:856-861.

14. Tran DM, Larsson $M$, Olson $L$ et al., High prevalence of colonisation with carbapenemresistant Enterobacteriaceae among patients admitted to Vietnamese hospitals: Risk factors 
and burden of disease, $\mathrm{J}$ Infect 2019; 3 https: //doi.org/10.1016/j.jinf.2019.05.013

15. Lowe CF, Kus JV, Salt N, et al. Nosocomial transmission of New Delhi Metallo- $\beta$-Lactamase-1producing Klebsiella pneumoniae in Toronto, Canada. Infect Control Hosp Epidemiol 2013; 34:49-55.

16. Jaiswal SR, Gupta S, Kumar RS, et al. Gut colonization with carbapenem-resistant Enterobacteriaceae adversely impacts the outcome in patients with hematological malignancies: results of a prospective surveillance study. Mediterr J Hematol Infect Dis 2018; 10(1): e2018025,

http://dx.doi.org/10.4084/MJHID.2018.025

17. Sandhu R, Aggarwal A, Sayal P, Kumar S. Intestinal carriage of drug-resistant Gram-negative bacteria belonging to family Enterobacteriaceae in children aged 3-14 years: An emerging threat. Int J Health Allied Sci 2019; 8:108-115. 